\title{
Role of total motile sperm count in the evaluation of young men with bilateral subclinical varicocele and asthenospermia
}

\author{
Georgios Tsampoukas 1, 2, 4, Athanasios Dellis 1,3, Antigoni Katsouri ${ }^{5}$, Dominic Brown ${ }^{2}$, \\ Konstantinos Deliveliotis ${ }^{6}$, Mohamad Moussa ${ }^{7}$, Noor Buchholz ${ }^{1}$, Athanasios Papatsoris ${ }^{1,6}$ \\ ${ }^{1}$ U-merge Ltd. (Urology for emerging countries), London, UK; \\ ${ }^{2}$ Department of Urology, Princess Alexandra Hospital, Harlow, UK; \\ ${ }^{3}$ Department of Urology, Aretaieion Academic Hospital, Athens, Greece; \\ ${ }^{4}$ Department of Urology, Agios Andreas Hospital, Patras, Greece; \\ ${ }^{5}$ Department of Pharmacy, University of Patras, Patras, Greece; \\ ${ }^{6} 2^{\text {nd }}$ Department of Urology, University Hospital of Athens, Athens, Greece; \\ ${ }^{7}$ Al Zahraa Hospital, University Medical Center, Lebanese University, Beirut, Lebanon. \\ *U-merge Ltd. (Urology for Emerging Countries) is an academic urological platform dedicated to facilitate knowledge transfer in urology on \\ all levels from developed to emerging countries. U-merge Ltd. is registered with the Companies House in London/ UK. www.U-merge.com
}

\section{Summary Introduction: In comparison to its clinical analogue, the subclinical varicocele repre-} sents a questionable entity and specific guidelines for the optimal management are lacking. In our previous study of patients with subclinical varicocele, we showed that bilateral condition is associated with risk of dyspermia. In the present study, we evaluated the risk of deterioration of semen quality in men with bilateral disease and impaired motility according to $\mathrm{WHO}$ criteria.

Materials and methods: Men with bilateral subclinical varicocele, not desiring fatherhood at the time of presentation, were included in study. During initial evaluation, the number of Total Motile Sperm Count (TMSC) was calculated and the patients' age, total testicular volume (TTV), maximum venous size and mean resistive index (RI) of the intratesticular arteries were recorded. We classified the participants in five classes according to the TMSC reading: class A-: TMSC $<5 \times 10^{6}$,

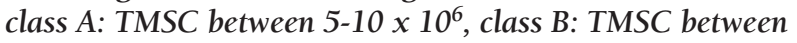

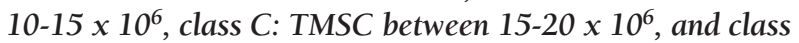
D: TMSC > $20 \times 10^{6}$ per ejaculate. The participants were seen after 6 months for a repeat spermiogram and physical examination. If clinical varicocele was diagnosed or a new abnormality in the spermiogram was noted, the participants were excluded from the study. The remaining patients were allocated to two groups according to the repeat TMSC reading: patients sub-classified into a lower class (group 1), and patients remaining at the same class (group 2). A comparative analysis was performed between two groups.

Results: Nineteen men were included. Nine patients were subclassified (group 1). Three patients moved to A- class (<5 $\left.x 10^{6}\right)$. Ten patients remained in the same class having no deterioration (group 2). Comparing the two groups, no statistically significant difference was recognized for age, TTV, maximum venous size on both sides, and mean RI ( $p>0.05)$. However, the initial reading for TMSC was $14.57 \times 10^{6}$ in group 1, and $22.84 \times 10^{6}$ in group 2 , respectively. This difference was statistically significant $(p<0.05)$. Additionally, in a paired analysis there was a significant difference in TMSC after 6 months $(p<0.05)$, too.
Conclusions: Young men with bilateral varicocele and asthenospermia seem to be at risk of deterioration in their semen quality after a follow-up of 6 months. The measurement of TMSC can unmask patients at risk, whereas men with the lowest readings seem to be at highest risk for deterioration. The possibility of a worsening sperm quality should be considered in the appropriate clinical context.

KEY WORDS: Varicocele; Subclinical; Total motile sperm count (TMSC); Infertility; Bilateral.

Submitted 21 June 2020; Accepted 24 August 2020

\section{INTRODUCTION}

A subclinical varicocele (SV) is defined as the radiological finding of a dilatation of the pampiniform plexus and the presence of venous reflux, in the absence of clinical varicocele $(\mathrm{CV})$ on physical examination $(1,2)$. The condition is regarded as an early stage for the development of a clinical varicocele and activity seems to increase the risk $(3,4)$. In comparison to its clinical analogue, a unilateral, subclinical varicocele is considered an entity of uncertain significance, and the evidence to support treatment, even in the context of male infertility, is weak (5).

Observation, surgical treatment, embolization and empirical treatment with clomiphene citrate or bioflavonoids has been proposed as possible management options (6-8). A special subgroup of infertile patients with right-sided subclinical and a simultaneous left-sided clinical varicocele seem to have a greater benefit from bilateral intervention in terms of improvement in semen quality and pregnancy rates in comparison to unilateral correction, implicating that the right subclinical varicocele is significant (9). In a similar clinical context, the presence of bilateral subclinical varicocele (BSV) seems to represent a distinct entity with noteworthy clinical significance. The prevalence of BSV seems to be higher in older men, whereas the condition is associated with deterioration of semen quality, especially sperm motility, during a long follow-up (10, 11). Previously, we had concluded that the presence of 
BSV has been associated with abnormal semen parameters in young men in comparison to unilateral SV (12). In this study, we followed a population of young men with BSV and asthenospermia in their spermiogram who did not desire fatherhood at time of presentation with a planned follow-up of 6 months. In order to evaluate their fertility capacity, we used the Total motile sperm count (TMSC) instead of classical WHO criteria. TMSC is considered a more reliable tool for the estimation of pregnancy when a male infertility factor is implicated (13). Our aim was to evaluate the risk of deterioration in semen quality and identify possible predictive factors in those patients.

\section{Materials AND METHOdS}

Inclusion criteria were males with bilateral subclinical varicocele, having asthenospermia alone $(<32 \%$ progressive motility) according to WHO 2010 criteria in their spermiogram and not desiring fatherhood at the time of presentation. The subjects were recruited during a period of 2 years (November 2016 - December 2018). Exclusion criteria were clinical varicocele, previous fatherhood, history of relevant surgery, present infertility concerns, active or chronic urogenital infections, signs and symptoms of primary hypogonadism, testicular microlithiasis, background of cancer, and history of intake of gonadotoxic medications or steroids. The subclinical varicocele was diagnosed by colour Doppler ultrasound (graded as grade I as per Hirsch classification) when the clinical examination was negative, but a venous dilatation larger than $2 \mathrm{~mm}$ of the pampiniform plexus with reflux during Valsava manoeuvre was demonstrated $(14,15)$. Testicular volume was measured by the ultrasound unit according to the formula volume $=0.53 \times$ length $\times$ width $\times$ height, and the mean value was obtained. Also, the mean resistive index (RI) of at least 3 intratesticular arteries on both sides, and the maximum vein diameter on each side were recorded. Asthenospermia was documented by two spermiograms, whereby the spermiogram with the lowest motility value was used for reference. As an objective tool for the assessment of sperm potential, the total motile sperm count was used: ejaculate volume $(\mathrm{V}) \mathrm{x}$ sperm concentration (SC) x progressive motility $(\mathrm{A}+\mathrm{B})$ divided by $100 \%$.

For the subsequent analysis, we allocated patients into five classes according to TMSC readings:

- Class A-: TMSC $<5 \times 10^{6}$

- Class A: TMSC 5-10 × $10^{6}$

- Class B: TMSC $10-15 \times 10^{6}$

- Class C: TMSC 15-20 x $10^{6}$

- Class D: TMSC > $20 \times 10^{6}$

The patients were seen again after six months with a repeat spermiogram and physical examination. According to those follow-up results, we then formed two groups:

- Group 1: patients reclassified into a lower category

- Group 2: patients without downclassification.

Patient who developed additional abnormalities (e.g. oligospermia) in the spermiogram, or evidence of clinical varicocele were excluded. The two groups were compared in terms of age, maximal left vein diameter, maximal right vein diameter, total testicular volume, mean RI value, the grade of reflux, initial TMSC, FSH and testosterone.

For statistical analysis, the Shapiro-Wilk test was used to check normality, and subsequently the Student's t-test and Mann-Whitney U-test were used accordingly for the detection of statistically significant differences between the two groups. Statistical significance value was set at a $p<0.05$. A Wilcoxon matched-pairs signed rank test was used to assess the difference in the TMSC at the first assessment, and at subsequent follow-up.

\section{RESULTS}

Nineteen patients were included in the study. The participants' characteristics are listed in Table 1.

The distribution of patients in classes initially, and at 6 months is depicted in Figure 1. Nine patients were downgraded into a lower class (group 1). Ten patients remained in the same class unchanged (group 2). Three patients downgraded from class A to class A- (TMSC $<5 \times 10^{6}$ ).

A paired t-test comparing the initial TMSC and the follow-up TMSC readings showed a significant difference after a mean follow-up of 6.7 months $(\mathrm{p}<0.05$, Table 2$)$. When comparing the mean values of all the parameters

\section{Figure 1.}

The classification of patients according to TMSC range:

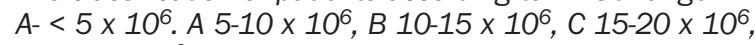
$D>20 \times 10^{6}$.

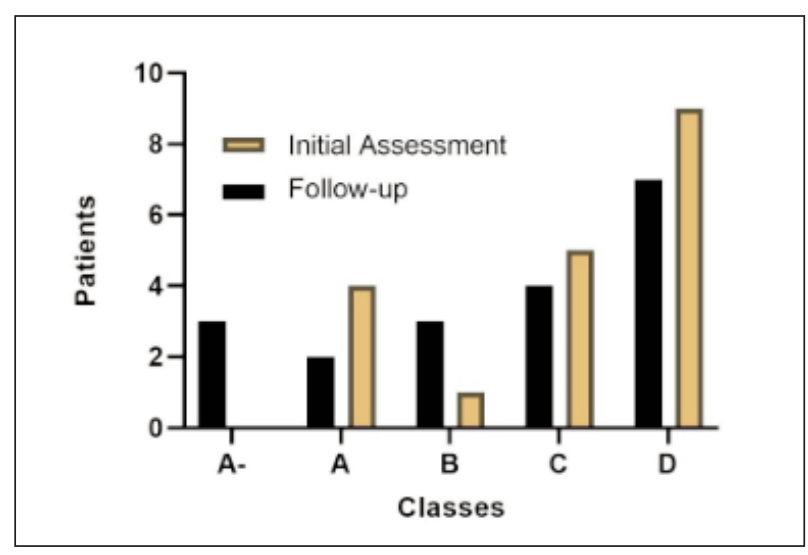

Table 1.

Patients' characteristics.

\begin{tabular}{|lcccccccc|}
\hline & $\begin{array}{c}\text { Age } \\
\text { (years) }\end{array}$ & $\begin{array}{c}\text { MVD } \\
\text { (left, } \mathbf{m m} \text { ) }\end{array}$ & $\begin{array}{c}\text { MVD } \\
\text { (right, } \mathbf{m m} \text { ) }\end{array}$ & $\begin{array}{c}\text { FU } \\
\text { (months) }\end{array}$ & RI & $\begin{array}{c}\text { Testosterone } \\
\text { (ng/dl) }\end{array}$ & $\begin{array}{c}\text { FSH } \\
\text { (mUl/ml) }\end{array}$ & $\begin{array}{c}\text { TV } \\
\text { (ml) }\end{array}$ \\
\hline No. & 19 & 19 & 19 & 19 & 19 & 19 & 19 & 19 \\
\hline Min. & 18 & 2.1 & 2.1 & 6 & 0.45 & 401 & 2 & 24.2 \\
\hline Max. & 34 & 3.2 & 2.8 & 8 & 0.64 & 762 & 7.5 & 37.8 \\
\hline Range & 16 & 1.1 & 0.7 & 2 & 0.19 & 361 & 5.5 & 13.6 \\
\hline Mean +/- SD & $26+4.22$ & $2.73+0.31$ & $2.56+0.17$ & $6.79+0.79$ & $0.555+0.05$ & $525+97.7$ & $3.83+1.31$ & $29.4+4.08$ \\
\hline Median & 26 & 2.80 & 2.60 & 7 & 0.55 & 489 & 3.90 & 28.1 \\
\hline Std. error of mean & 0.967 & 0.072 & 0.04 & 0.181 & 0.012 & 22.4 & 0.3 & 0.936 \\
\hline SD: Standard Deviation, MVD: maximal vein diameter, FU: Follow-up in months, Rl: Resistive index, FSH: Follicle-stimulating hormone, TV: total testicular volume. \\
\hline
\end{tabular}


Table 2.

Match-up comparison of TMSC after mean follow-up of 6.7 months.

\begin{tabular}{|c|c|c|c|}
\hline & ITMSC (millions) & fTMSC (millions) & Wilcoxon test \\
\hline No. & 19 & 19 & \\
\hline$\overline{\text { Min. }}$ & 6,32 & 4,22 & \\
\hline Max. & 28,6 & 28.2 & \\
\hline Range & 22.2 & 24 & \\
\hline$\overline{M e a n+/-S D}$ & $18.9+7.32$ & $16.5+8.13$ & \\
\hline$\overline{\text { Median }}$ & 20 & 16.8 & \\
\hline Std. error of mean & 1.68 & 1.86 & \\
\hline Pvalue & & & $0.0002^{*}$ \\
\hline \multicolumn{4}{|c|}{$\begin{array}{l}\text { *Difference statistically significant, } p<0.05 \text {, Number of pairs } 19 \text {, Sum of positive ranks } 10.50 \text {, Sum of negative } \\
\text { ranks - 179.5, two-tailed, Median of differences -3.0, iTMSC: initial Total motile sperm count, fTMSC: follow-up total } \\
\text { motile sperm count. }\end{array}$} \\
\hline
\end{tabular}

Table 3.

Comparison of parameters between Group 1 and Group 2.

\begin{tabular}{|c|c|c|c|c|c|c|}
\hline Parameter & Group & No. & Mean & T-test & U-value & $P$ value \\
\hline \multirow[t]{2}{*}{ Age (years) } & Group 1 & 9 & 25.56 & $\mathrm{t}=0.08748, \mathrm{df}=17$ & & 0.93 \\
\hline & Group 2 & 10 & 25.40 & & & \\
\hline \multirow[t]{2}{*}{$\overline{\text { MVD (right, mm) }}$} & Group 1 & 9 & 2.622 & $\mathrm{t}=1.447, \mathrm{df}=17$ & & 0.16 \\
\hline & Group 2 & 10 & 2.51 & & & \\
\hline \multirow[t]{2}{*}{ iTMSC $\left(1 \times 10^{6}\right)$} & Group 1 & 9 & 14.57 & $\mathrm{t}=2.930, \mathrm{df}=17$ & & $0.0093^{*}$ \\
\hline & Group 2 & 10 & 22.84 & & & \\
\hline \multirow[t]{2}{*}{ RI } & Group 1 & 9 & 0.57 & $t=1.116, d f=17$ & & 0.27 \\
\hline & Group 2 & 10 & 0.54 & & & \\
\hline \multirow[t]{2}{*}{ Testosterone (ng/dl) } & Group 1 & 9 & 545.3 & $\mathrm{t}=0.8542, \mathrm{df}=17$ & & 0.40 \\
\hline & Group 2 & 10 & 506.7 & & & \\
\hline \multirow[t]{2}{*}{ FSH (mUl/ml) } & Group 1 & 9 & 3.22 & $t=2.082, d f=17$ & & 0.052 \\
\hline & Group 2 & 10 & 4.37 & & & \\
\hline \multirow[t]{2}{*}{$\overline{\pi V}(\mathrm{ml})$} & Group 1 & 9 & 27.69 & & 38 & 0.58 \\
\hline & Group 2 & 10 & 29.97 & & & \\
\hline \multirow[t]{2}{*}{ MVD (Left, mm) } & Group 1 & 9 & 2.8 & & 40.50 & 0.73 \\
\hline & Group 2 & 10 & 2.85 & & & \\
\hline
\end{tabular}

in both groups, no significant difference was detected in terms of age, maximal vein diameter on both sides, RI, testosterone, FSH and TTV. The initial TSMC reading was however significantly different between the patients in both groups $(\mathrm{p}<0.05)$ (Table 3$)$.

\section{Discussion}

We decided to study men with bilateral varicocele as we regard the condition as the full expression of varicocele disease. The prevalence has been reported as up to $80 \%$, and differences in detection rates are the result of differences in diagnostic approach $(16,17)$. Moreover, bilateral varicocelectomy is superior to unilateral in terms of the main outcome of pregnancy rates $(9,18)$.

Furthermore, the possible common pathophysiological background (BSV and asthenospermia) and similar expectations (no fertility issues and no desire for fatherhood at that time) formed a homogenous group which gives reproducibility to our results.

We used TMSC as a marker of semen quality as it is a superior predictive tool for the main outcomes for male infertility in comparison to standard WHO criteria. Specifically, the well-studied range classification into 3 groups (TMSC $<5 \times 10^{6} ; 5-20 \times 10^{6} ;>20 \times 10^{6}$ spermatozoa, regarded as normospermia) seems to have a superior predictive value in terms of spontaneous pregnancy rates whereas the same applies for infertile couples with male factor infertility undergoing intracytoplasmic sperm injection cycles (ICSI) $(19,20)$. Also, TMSC can be used as a method to assess the clinical outcome of varicocelectomy in patients with clinical varicocele (CV), and predict the need of further assisted reproduction technology treatment (21). Additionally, a study found no significant differences in the improvement of TMSC after repair in men with SV comparing to men with CV whereas most of patients in the subclinical group had BSV which is relevant to our study (22). In our cohort, the paired analysis showed a significant difference between the initial and follow-up TMSC readings. This means that this group of men might be at risk of deterioration of sperm motility in the future. Also, we observed that those men experiencing deterioration had their readings below the so-called normal cut-off of $20 \times 10^{6}(14.57 \mathrm{x}$ $10^{6}$ vs $\left.22.84 \times 10^{6}, \mathrm{p}<0.05\right)$. Moreover, 3 out of 4 patients with an initial TMSC range of $5-10 \times 10^{6}$ were later found with a TMSC range of $<5 \times 10^{6}$ at follow-up. Although these readings do not necessarily imply infertility, this range is nevertheless associated with lower chances of spontaneous pregnancy rates. In clinical practice, infertile patients with these readings might have been advised to undergo assisted-reproductive modalities (19). Thus, our results imply that patients with BSV and asthenospermia with gray-zone values of TMSC $<20$ $\mathrm{x} 10^{6}$, and especially those with the lowest readings may be at risk of deterioration of their semen quality.

No statistically significant difference was found between the two groups in terms of age and no specific conclusions can be drawn. Once again, the results must be linked to the appropriate clinical context, e.g. the perspective of the patients and the possible expectations of the couple. Age does not seem to be a decisive factor for dyspermia in patients with SV (23), but it does have importance in patients with low grade varicocele undergoing varicocelectomy in relation to actual venous size (24). Also, we examined the role of vein diameter on both sides and there was no significant difference between the groups. These results are in accordance with our previous study where no association was found between maximal vein diameter in men with SV and dyspermia (12). This does not sound surprising, as even in clinical forms, even grade seems to be inferior to reflux in terms of prediction of the results of varicocelectomy (25). In our study, the role of reflux was not exhaustively examined, as all of our participants by definition had Grade I varicocele (Valsava induced reflux) according to Hirsch classification, which is helpful for the diagnosis of the subclinical form but lacks pathophysiological significance in adults (26).

In young boys, pattern of grade I reflux helps stratify the risk for hypotrophy and assist the follow-up (27). In our previous study in adult men, the pattern of the reflux (lasting all the duration of the Valsava vs short-lasting reflux) had not been linked to dyspermia in adults patients with subclinical varicocele (12). Reflux is note- 
worthy though, and the shunt-type (continuous) reflux is strongly associated with testicular hypotrophy in adolescents (28), whereas prolonged reflux may also predict the outcome of varicocelectomy in adults (25). In terms of the endocrinological profile in our study participants, there was no difference between groups in testosterone or FSH. Additionally, all readings were within normal limits reflecting the normal testicular volume found in both groups. It is doubtful if SV should be expected to have any significant effect on testicular volume, and the reports are conflicting (29-31).

Furthermore, we examined the role of intratesticular RI at the cut-off of 0.6 which has been highlighted as a marker of dyspermia $(32,33)$. We did not focus on linked parameters Peak Systolic Velocity (PSV) and End Diastolic Velocity (EDV) as RI is not associated with the angle of recording (which is extremely demanding in tiny arteries like intratesticular ones) and, therefore, operator-independent and more reliable for the scope of our study. In our cohort, there was no difference between the two groups in terms of RI, whereas the median value in our sample could be considered as normal. This discrepancy might be reflected by the pathophysiological background. Varicocele may provoke damage in various sites in the genital tract apart from the testicle e.g. the epididymis which is vital for the motility potential of the spermatozoa and some agents are under evaluation (34-36). Even low-grade varicoceles may undermine epididymal function causing infertility issues which might be apparent even when the spermiogram is normal (37). Another intriguing aspect lies on the intercommunication between the pampiniform and the periprostatic plexus $(38,39)$. In the varicoceleassociated infertility, patients with deteriorated motility undergoing varicocelectomy might not experience improvement if the periprostatic plexus is apparently dilated which implicates the complexity in the pathophysiology of the condition (40). Also, the topography of the damage caused by the SV might be the reason why infertile patients with SV have low levels of oxidative stress markers (41). In our study, the participants had normal testicles, normal RI and asthenospermia; it could be assumed that if BSV was responsible for the semen deterioration, it might have caused damage in a site different than the testicles and this is why RI is within normal limits. Either way, it is doubtful if RI can assist during the evaluation of men with SV.

We appreciate our study has certain limitations. First and foremost, the men involved had no fertility concerns at that time and they must not be considered infertile. Also, we did not correlate with pregnancy rates, which is the main outcome measure in the evaluation of infertile patients with varicocele and therefore, our study cannot provide safe conclusions to infertile patients with the condition. The clinical merit of our findings must be set in an appropriate clinical context of men incidentally found with bilateral SVs. Secondly, we appreciate that our sample is small. However, the presentation and the selection criteria were major limitations because as matter of fact, the participants represented an asymptomatic population who normally do not attend in the hospital. We managed to increase the sample size through screening and by inviting young men to the cohort.

\section{Conclusions}

Despite our small sample, our results showed that young males with BSV and asthenospermia are likely to experience deterioration in their TMSC over time. Importantly, patients with borderline TMSC readings seem to be at higher risk of deterioration, and especially patients with very low readings.

Thus, these patients might not be ideal candidates for a wait $\&$ watch approach although the optimal management is unknown. Of note, there was no correlation with pregnancy rates and therefore, our observations must be utilized in the appropriate clinical context.

\section{REFERENCES}

1. Belay R, Huang G, Shen J.-C., Ko EK. Diagnosis of clinical and subclinical varicocele: how has it evolved? Asian J Androl. 2016; 18:182.

2. Patil V, Shetty SMCC. Das S Redefining the criteria for grading varicoceles based on reflux times: A clinicoradiological correlation. Ultrasound Q. 2016; 32:82-85.

3. Cervellione RM, Corroppolo M, Bianchi A. Subclinical varicocele in the pediatric age group. J Urol. 2008; 179:717-9.

4. Zampieri N, Dall'Agnola A. Subclinical varicocele and sports: A longitudinal study. Urology 2011; 77:1199-1203.

5. Jungwirth A, Diemer T, Kopa Z, Male infertility. EAU guidelines. 2016.

6. Seo JT, Kim KT, Moon MH, Kim WT. The significance of microsurgical varicocelectomy in the treatment of subclinical varicocele. Fertil. Steril. 2010; 93:1907-1910.

7. Unal D, Yeni E, Verit A, Karatas OF. Clomiphene citrate versus varicocelectomy in treatment of subclinical varicocele: A prospective randomized study. Int J Urol. 2001; 8:227-230.

8. Zampieri N, Pellegrino M. Effects of bioflavonoids in the management of subclinical varicocele Pediatr Surg Int. 2010; 26:505-8.

9. Ou N, Zhu J, Zhang W, et al. Bilateral is superior to unilateral varicocelectomy in infertile men with bilateral varicocele: Systematic review and meta-analysis. Andrologia. 2019;51:e13462.

10. Chen SSS. Significant predictive factors for subfertility in patients with subclinical varicocele. Andrologia. 2017; 49:1-5.

11. Hallak J. Asymptomatic male currently not desiring fertility with bilateral subclinical varicocele found on ultrasound evaluation and borderline semen analysis results. Asian J Androl. 2016; 18:315.

12. Tsampoukas G, Dellis A, Papatsoris A. Bilateral disease and intratesticular haemodynamics as markers of dyspermia in patients with subclinical varicocele: A prospective study. Arab J Urol. 2019; 17:298-304

13. Borges EJ. Total motile sperm count: a better way to rate the severity of male factor infertility? JBRA Assist Reprod. 2016; 20:47-8.

14. Hirsh AV, Cameron KM, Tyler JP, et al. The Doppler assessment of varicoceles and internal spermatic vein reflux in infertile men. $\mathrm{Br}$ J Urol. 1980; 52:50-56.

15. Gonda RL, Karo JJ, Forte RA, O'Donnell KT. Diagnosis of subclinical varicocele in infertility. Am J Roentgenol. 1987; 148:71-75.

16. Gat Y, Bachar GN, Zukerman Z, et al. Varicocele: a bilateral disease. Fertil. Steril. 2004; 81:424-429.

17. Gat Y, Bachar GN, Zukerman Z,, et al. Physical examination 
may miss the diagnosis of bilateral varicocele: a comparative study of 4 diagnostic modalities. J Urol. 2004; 172:1414-1417.

18. Donkol RH, Salem T. Paternity after varicocelectomy: preoperative sonographic parameters of success. J Ultrasound Med. 2007; 26:593-9.

19. Hamilton JA, Cissen M, Brandes M, et al. Total motile sperm count: a better indicator for the severity of male factor infertility than the WHO sperm classification system. Hum Reprod. 2015; 30:1110-21.

20. Borges E Jr, Setti AS, Braga DP, et al. Total motile sperm count has a superior predictive value over the WHO 2010 cut-off values for the outcomes of intracytoplasmic sperm injection cycles. Andrology. 2016; 4:880-6.

21. Samplaski MK, Lo KC, Grober ED, et al. Varicocelectomy to 'upgrade' semen quality to allow couples to use less invasive forms of assisted reproductive technology. Fertil Steril. 2017; 108:609-612.

22. Thirumavalavan N, Scovell JM, Balasubramanian A, et al. The Impact of Microsurgical Repair of Subclinical and Clinical Varicoceles on Total Motile Sperm Count: Is There a Difference? Urology. 2018; 120:109-113.

23. Chen SS. Significant predictive factors for subfertility in patients with subclinical varicocele. Andrologia 2017; 49(10).

24. Shiraishi K, Takihara H, Naito K. Internal spermatic vein diameter and age at operation reflect the response to varicocelectomy Andrologia. 2001; 33:351-5.

25. Goren MR, Erbay G, Ozer C, et al. Can we predict the outcome of varicocelectomy based on the duration of venous reflux? Urology. 2016; 88:81-86.

26. Freeman S, Bertolotto M, Richenberg J, et al. Ultrasound evaluation of varicoceles: guidelines and recommendations of the European Society of Urogenital Radiology Scrotal and Penile Imaging Working Group (ESUR-SPIWG) for detection, classification, and grading. Eur Radiol. 2020; 30:11-25.

27. Zampieri N, Zuin V, Corroppolo M, et al. Relationship between varicocele grade, vein reflux and testicular growth arrest. Pediatr Surg Int. 2008; 24:727-30.

28. Mohseni MJ, Nazari H, Amini E, et al. Shunt-type and stop-type varicocele in adolescents: prognostic value of these two different hemodynamic patterns. Fertil Steril. 2011; 96:1091-6.

29. Sakamoto H, Ogawa Y, Yoshida H. Relationship between testicular volume and varicocele in patients with infertility. Urology. 2008; 71:104-109.

30. Akcar N, Turgut M, Adapinar B, Ozkan IR. Intratesticular arterial resistance and testicular volume in infertile men with subclinical varicocele. J Clin Ultrasound. 2004; 32:389-393.

31. Zini A, Buckspan M, Berardinucci D, Jarvi K. The influence of clinical and subclinical varicocele on testicular volume. Fertil Steril. 1997; 68:671-674.

32. Pinggera GM, Mitterberger M, Bartsch G, et al. Assessment of the intratesticular resistive index by colour Doppler ultrasonography measurements as a predictor of spermatogenesis. BJU Int. 2008; 101:722-6.

33. Hillelsohn JH,Chuang KW, Goldenberg E, Gilbert BR. Spectral doppler sonography: A noninvasive method for predicting dyspermia. J Ultrasound Med. 2013; 32:1427-1432.

34. Akdemir S, Gurocak S, Konac E, et al. Different surgical techniques and L-carnitine supplementation in an experimental varicocele model. Andrologia. 2014; 46:910-6.
35. Milingos SD. The epidymal factor - a diagnostic approach. Int J Androl. 1985; 8:417-420.

36. Mongioi L, Calogero AE, Vicari E, et al. The role of carnitine in male infertility. Andrology. 2016; 4:800-7.

37. Pajovic B, Dimitrovski A, Radojevic N, Vukovic M. A correlation between selenium and carnitine levels with hypo-osmotic swelling test for sperm membrane in low-grade varicocele patients. Eur Rev Med Pharmacol Sci. 2016; 20:598-604.

38. De Caestecker K, Lumen N, Spinoit AF, et al. Varicocele: the origin of benign prostatic hypertrophy? Testosterone dosages in the periprostatic plexus. Acta Clin Belg. 2016; 71:281-283.

39. Gat Y, Goren M. Benign Prostatic Hyperplasia: Long-term follow-up of prostate volume reduction after sclerotherapy of the internal spermatic veins. Andrologia. 2018; 50.

40. Condorelli RA, Calogero AE, Mongioi' L, et al. Varicocele and concomitant dilation of the periprostatic venous plexus: effects on semen viscosity sperm parameters. J Endocrinol Invest. 2016; 39:543-7.

41. Ni K, Steger K, Yang H, et al. A comprehensive investigation of sperm DNA damage and oxidative stress injury in infertile patients with subclinical, normozoospermic, and astheno/oligozoospermic clinical varicocoele. Andrology. 2016; 4:816-24.

\section{Correspondence}

Georgios Tsampoukas, MD (Corresponding Author)

tsampoukasg@gmail.com

scientific-office@u-merge.com

Noor Buchholz, MD

noor.buchholz@gmail.com

U-merge Scientific Office

1, Menandrou Street, Athens 14561 (Greece)

Dominic Brown, MD

dominic.brown5@nhs.net

Department of Urology, Princess Alexandra Hospital, Harlow (UK)

Athanasios Dellis, MD

aedellis@gmail.com

Department of Urology, Aretaieion Academic Hospital, Athens (Greece)

Antigoni Katsouri, MD

a.katsr@gmail.com

Department of Pharmacy, University of Patras, Patras (Greece)

Konstantinos Deliveliotis, MD

chdeliveli@gmail.com

Athanasios Papatsoris, MD

agpapatsoris@yahoo.gr

2nd Department of Urology, University Hospital of Athens, Athens (Greece)

Mohamad Moussa, MD

mohamad.moussa@zhumc.org.lb

Al Zahraa Hospital, University Medical Center, Lebanese University, Beirut (Lebanon) 